\title{
Modular Leech Trees of Order at Most 8
}

\author{
David Leach \\ Department of Mathematics, University of West Georgia, 1601 Maple Street, Carrollton, GA 30118, USA \\ Correspondence should be addressed to David Leach; cleach@westga.edu
}

Received 11 August 2014; Accepted 2 September 2014; Published 18 September 2014

Academic Editor: Laszlo A. Szekely

Copyright (C) 2014 David Leach. This is an open access article distributed under the Creative Commons Attribution License, which permits unrestricted use, distribution, and reproduction in any medium, provided the original work is properly cited.

In 1975, John Leech asked when can the edges of a tree on $n$ vertices be labeled with positive integers such that the sums along the paths are exactly the integers $1,2, \ldots,\left(\begin{array}{c}n \\ 2\end{array}\right)$. He found five such trees, and no additional trees have been discovered since. In 2011 Leach and Walsh introduced the idea of labeling trees with elements of the group $\mathbb{Z}_{k}$ where $k=\left(\begin{array}{c}n \\ 2\end{array}\right)+1$ and examined the cases for $n \leq 6$. In this paper we show that no modular Leech trees of order 7 exist, and we find all modular Leech trees of order 8.

\section{Introduction}

A tree on $n$ vertices is said to be a Leech tree if its edges can be weighted with positive integers in such a way that each of the $\left(\begin{array}{c}n \\ 2\end{array}\right)$ paths has a distinct weight from the set $\left\{1,2, \ldots,\left(\begin{array}{c}n \\ 2\end{array}\right)\right\}$. The weight of a path is found by summing all of its edge weights. Leech [1] found the five examples shown in Figure 1, which are to date the only ones known. In 1977, Taylor [2] proved that, in order for a Leech tree of order $n$ to exist, it must be that $n=k^{2}$ or $n=k^{2}+2$ for some integer $k$. Since then it has been shown by several authors [3-5] that no Leech trees of order 9,11 , or 16 exist, leaving $n=18$ as the smallest open case. Székely et al. [4] have conjectured that no additional Leech trees exist. Since Leech trees are so difficult to come by, we consider the generalization to modular Leech trees.

Let $T$ be a tree on $n$ vertices and let $k=\left(\begin{array}{l}n \\ 2\end{array}\right)+1$. We say that $T$ is a modular Leech tree if there exists an edge weighting function $w: E(T) \rightarrow \mathbb{Z}_{k}$ such that each of the $\left(\begin{array}{l}n \\ 2\end{array}\right)$ paths within $T$ has a distinct weight from $1,2, \ldots,\left(\begin{array}{c}n \\ 2\end{array}\right)$ with the sums taken modulo $\left(\begin{array}{l}n \\ 2\end{array}\right)+1$. We call such an edge weighting function a $\mathbb{Z}_{k}$-Leech labeling. Since the pathweights are all distinct, the function $w$ induces a bijection between the paths of $T$ and the elements of the group $\mathbb{Z}_{k}$. We use $w$ to refer to this bijection as well.

Note that a "normal" Leech tree of order $n$ is also a modular Leech tree over $\mathbb{Z}_{\left(\begin{array}{c}n \\ 2\end{array}\right)+1}$ in which none of the path sums, before applying the mod operation, have a weight greater than $\left(\begin{array}{c}n \\ 2\end{array}\right)$. Thus Leech's original five examples provide us with five modular Leech trees. The only other example previously known is the tree of order 6 found in [6] shown in Figure 2. It was also shown in [6] that no modular Leech tree of order 5 exists.

In Section 2 we will see how Taylor's condition applies to modular Leech trees, and in Section 3 we will enumerate all Leech trees of order at most 8.

\section{Taylor's Condition for Modular Leech Trees}

For the normal Leech trees, Taylor's condition restricts the possible orders severely. However, with modular Leech trees over $\mathbb{Z}_{k}$, Taylor's condition only applies when $k$ is even. The proof is very similar to half of Taylor's proof.

Theorem 1. Suppose that $T$ is a modular Leech tree of order $n$ and $n \equiv 2$ or $3(\bmod 4)$; then $n=m^{2}+2$ for some integer $m$.

Proof. Assume that $T$ is a modular Leech tree of order $n$ and that $n \equiv 2$ or $3(\bmod 4)$. Since $n \equiv 2$ or $3(\bmod 4)$, we have that $\left(\begin{array}{l}n \\ 2\end{array}\right)$ is odd and thus the modulus $\left(\begin{array}{l}n \\ 2\end{array}\right)+1$ is even.

We color each vertex of the tree black or white as follows: start at any vertex $v$ and color it black. From $v$ we color all other vertices by traversing the edges of the graph. We keep the same color across edges with even weight and change colors across edges with odd weight. When all vertices are colored, an edge connects different colored vertices if and only if its weight is odd. Furthermore, since the modulus is even, for any vertices $u, v \in V(T)$, the path from $u$ to $v$ has 


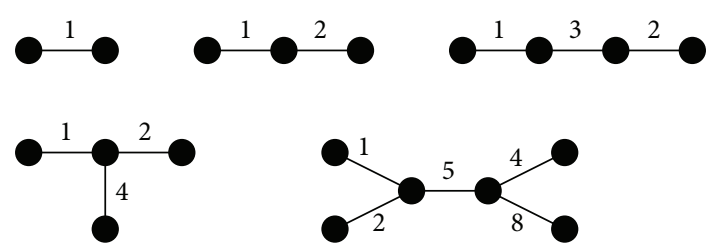

FIgURE 1: The five known Leech trees.

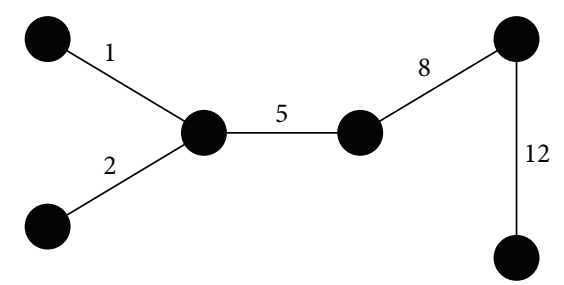

FIGURE 2: Leech tree of order 6 over $\mathbb{Z}_{16}$.

odd weight if and only if $u$ and $v$ are colored with opposite colors.

Now we count the number of odd paths in two ways: let $b$ and $w$ be the number of black and white vertices, respectively. Thus the number of odd paths is $b w$. Also, since there are $\left(\begin{array}{c}n \\ 2\end{array}\right)$ paths and $\left(\begin{array}{c}n \\ 2\end{array}\right)$ is odd, the number of odd paths is $(1 / 2)((n(n-$ $1) / 2)+1)$. Putting these together gives $n-2=n^{2}-4 b w$. Substituting $b+w$ for $n$ on the right side leads to $n=(b-w)^{2}+2$ and the theorem is proved.

The proof of Theorem 1 makes use of the fact that, under the assumptions of the theorem, a path has odd weight if and only if it contains an odd number of odd-weight edges. This fact does not hold when the modulus is odd. (For example, consider a path on four vertices with edges weighted 3,5, and 1 and examine the path-weights mod 7.) Recall that a normal Leech tree on $n$ vertices is also a modular Leech tree over $\mathbb{Z}_{k}$ where $k=\left(\begin{array}{c}n \\ 2\end{array}\right)+1$ and by Taylor's condition $n=m^{2}$ or $m^{2}+2$ for some integer $m$. We can conclude the following.

Theorem 2. If there exists a modular Leech tree of order $n$, and $n$ is not $m^{2}+2$ for some integer $m$, then $n \equiv 0$ or $1(\bmod 4)$.

\section{Computational Results}

We have already seen modular Leech trees of orders 2, 3, 4, and 6. By Leach and Walsh [6] and Theorem 2 we know that none exist for 5 or 7 . To examine larger values of $n$, we use computer search. The following theorem and corollary reduce the space that must be searched.

Theorem 3. Let $n$ be an integer and $k=\left(\begin{array}{c}n \\ 2\end{array}\right)+1$. Suppose that $f: E(T) \rightarrow \mathbb{Z}_{k}$ is a $\mathbb{Z}_{k}$-Leech labeling of a tree $T$. Then for any $b \in \mathbb{Z}_{k}$ satisfying $\operatorname{gcd}(b, k)=1$, the function $g: E(T) \rightarrow \mathbb{Z}_{k}$ defined by $g(e)=b \cdot f(e)$ is also a $\mathbb{Z}_{k}$-Leech labeling of $T$.

Proof. Let $P(T)$ be the set of all paths in $T$. We can consider $f$ as a bijection between $P(T)$ and $\mathbb{Z}_{k}$, so for every nonzero

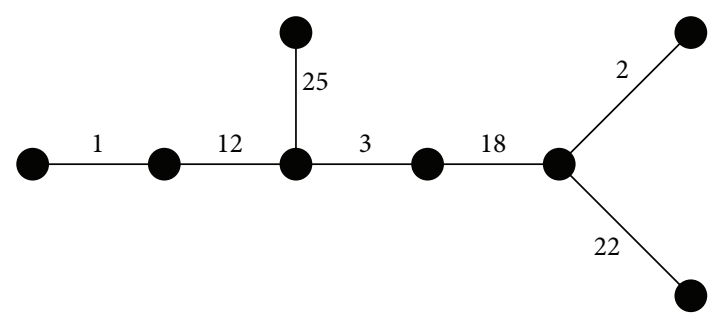

FIGURE 3: Leech tree of order 8 over $\mathbb{Z}_{29}$.

$b \in \mathbb{Z}_{k}$, there exists a path with weight $b$. Now define $g$ : $P(T) \rightarrow \mathbb{Z}_{k}$ by $g(e)=b \cdot f(e)$.

We now show that $g: P(T) \rightarrow \mathbb{Z}_{k}$ is bijective: let $P_{1}$ and $P_{2}$ be paths in $T$ and suppose that $g\left(P_{1}\right)=g\left(P_{2}\right)$. Then $b \cdot f\left(P_{1}\right)=b \cdot f\left(P_{1}\right)$. Since $g c d(b, k)=1, b^{-1}$ exists and thus $f\left(P_{1}\right)=f\left(P_{2}\right)$. Since $f$ is a bijective, $P_{1}=P_{2}$ and thus $g$ is also bijective. Since $g: P(T) \rightarrow \mathbb{Z}_{k}$ is bijective, $g$ is a Leech labeling of $T$.

Corollary 4. If there exists a $\mathbb{Z}_{k}$-Leech labeling of a tree $T$ and $e \in E(T)$, then there exists a $\mathbb{Z}_{k}$-Leech labeling of $T$ in which edge e has weight 1 .

For $n=8$, there are 23 distinct unlabeled trees. By computer search, we find that there is one modular Leech tree for $n=8$ and the edge-weighting function is unique, up to group and graph isomorphism. It is shown in Figure 3.

\section{Conflict of Interests}

The author declares that there is no conflict of interests regarding the publication of this paper.

\section{References}

[1] J. Leech, "Research problems: another tree labelling problem," The American Mathematical Monthly, vol. 82, no. 9, pp. 923-925, 1975.

[2] H. Taylor, "Odd path sums in an edge-labeled tree," Mathematics Magazine, vol. 50, no. 5, pp. 258-259, 1977.

[3] H. Taylor, "A distinct distance set of 9 nodes in a tree of diameter 36," Discrete Mathematics, vol. 93, no. 2-3, pp. 167-168, 1991.

[4] L. A. Székely, H. Wang, and Y. Zhang, "Some non-existence results on Leech trees," Bulletin of the Institute of Combinatorics and its Applications, vol. 44, pp. 37-45, 2005.

[5] B. Calhoun, K. Ferland, L. Lister, and J. Polhill, "Minimal distinct distance trees," Journal of Combinatorial Mathematics and Combinatorial Computing, vol. 61, pp. 33-57, 2007.

[6] D. Leach and M. Walsh, "Generalized Leech trees," Journal of Combinatorial Mathematics and Combinatorial Computing, vol. 78, pp. 15-22, 2011. 


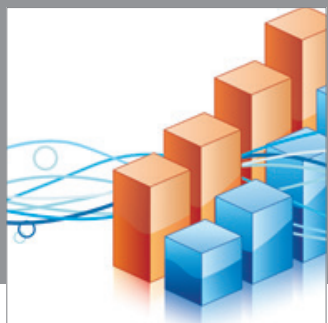

Advances in

Operations Research

mansans

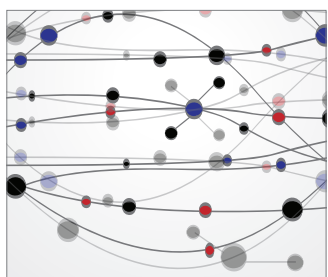

The Scientific World Journal
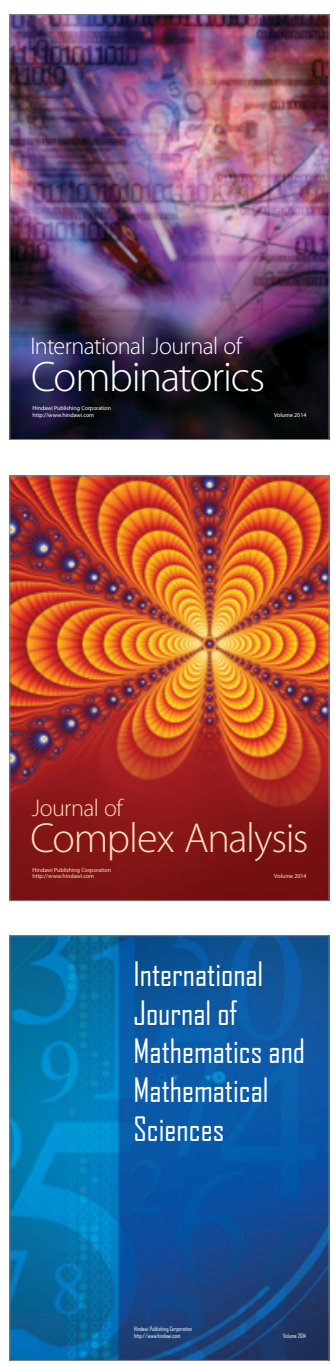
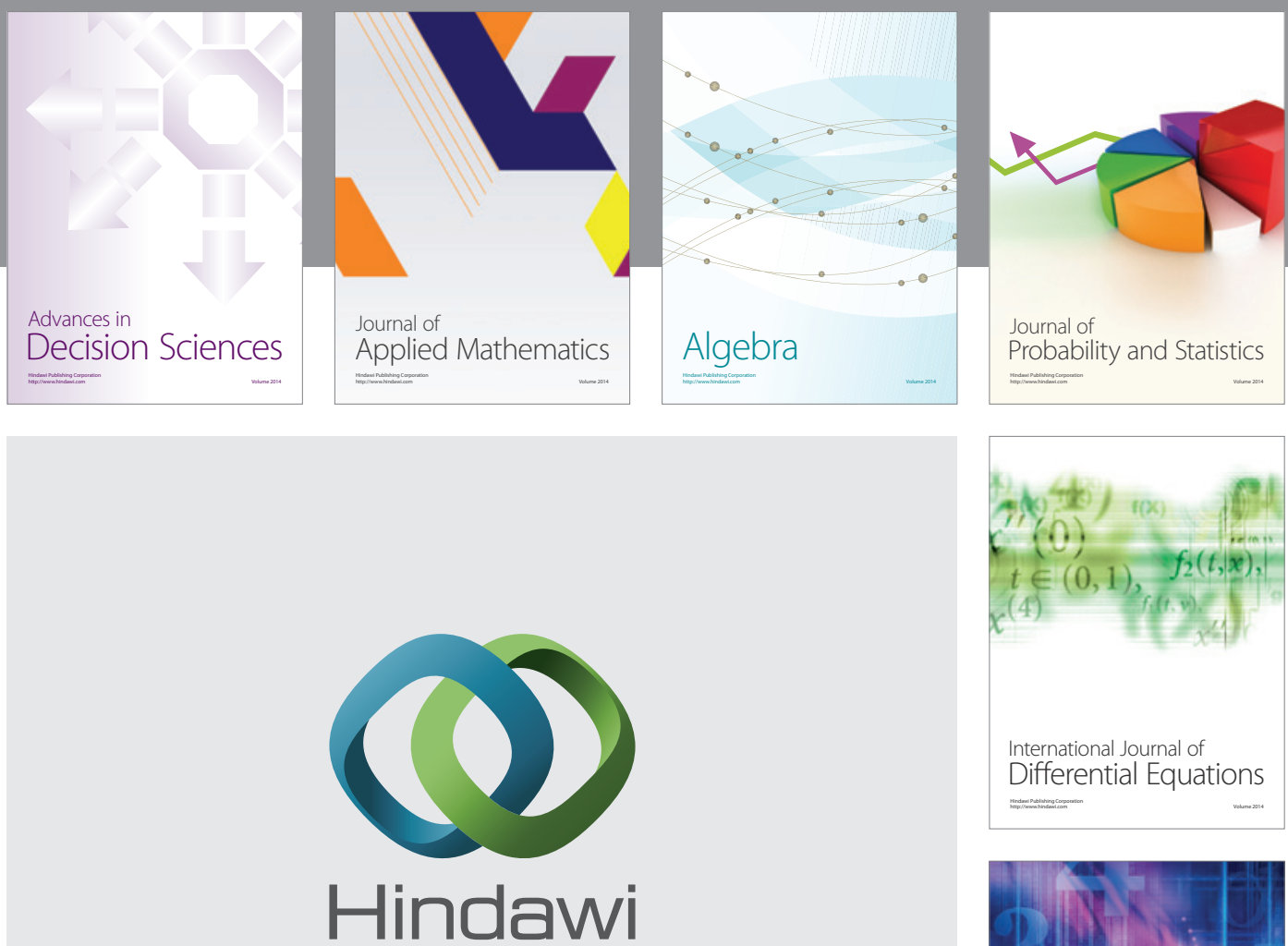

Submit your manuscripts at http://www.hindawi.com
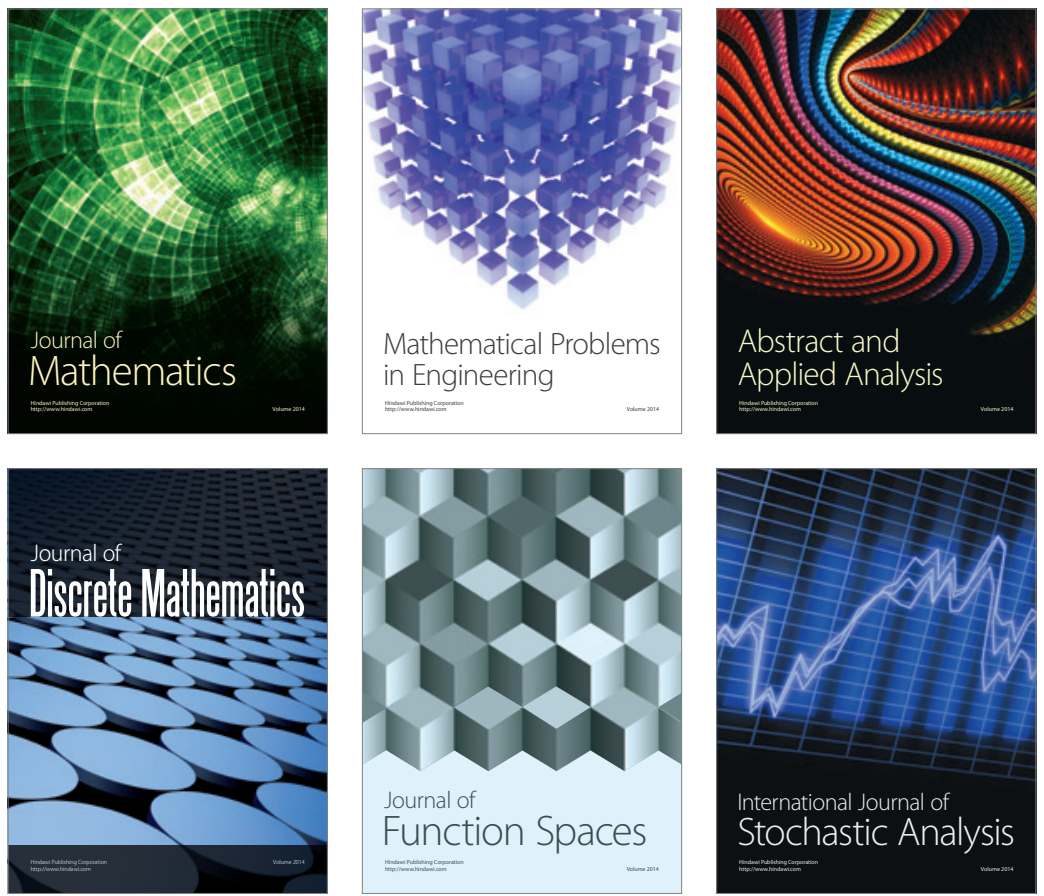

Journal of

Function Spaces

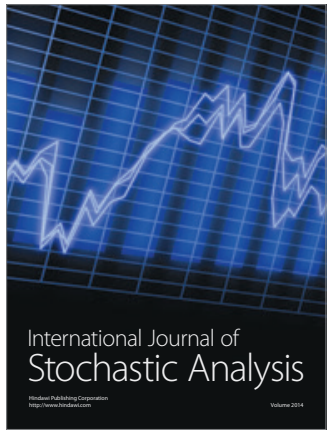

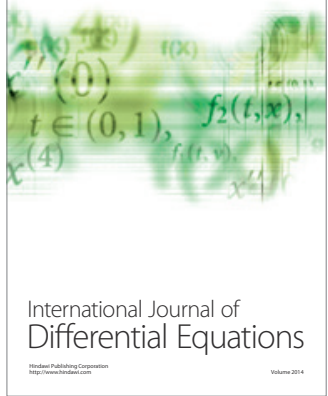
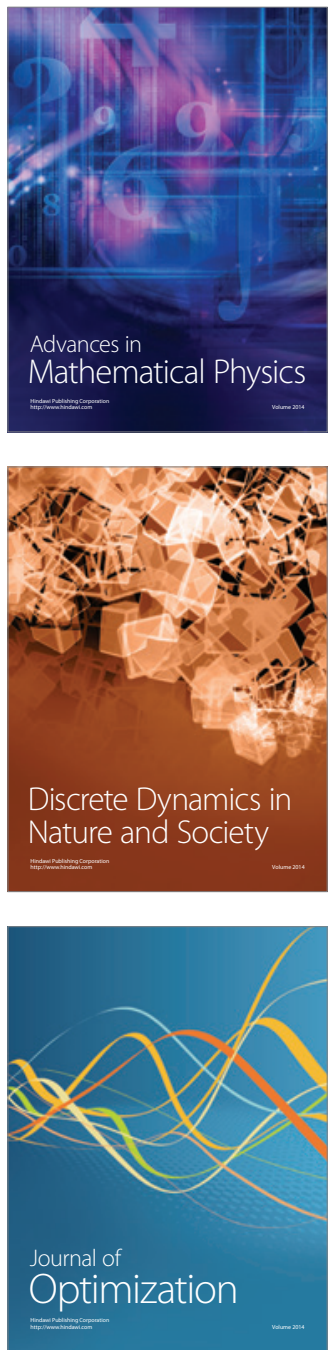